\title{
Nanoscale Friction Behaviors of Hierarchical Superhydrophobic Structure of Diamond-like Carbon Films with Various Humidity Conditions*
}

\author{
Young-Jun JANG**, Hiroyuki KOUSAKA** and Noritsugu UMEHARA** \\ ** Department of Mechanical Science and Engineering, Graduate School of Engineering, \\ Nagoya University, Furo-cho, Chikusa-ku, Nagoya, Aichi 464-8603, Japan \\ E-mail:jang@ume.mech.nagoya-u.ac.jp
}

\begin{abstract}
Superhydrophobic double roughening structure of DLC film was prepared by 2.45 $\mathrm{GHz}$ surface wave-excited plasma CVD with the mixture of methane $\left(\mathrm{CH}_{4}\right)$ and tetramethylsilane (TMS: $\left.\mathrm{Si}\left(\mathrm{CH}_{3}\right)_{4}\right)$ gases on the undulated DLC film by a series of plasma Ar etching, coating process and plasma Ar etching. Static wetting angle of water was observed that double roughening structure of DLC was superhydrophobicity such as wetting angle $161^{\circ}$. This approach also increased in air pockets easily trap among the needle-like posts. For the low friction at nanoscale, the surface wettability of the solid lubrication played a significant role, when the DLC film modified from flat to double roughening structure, the friction was constantly inner humidity conditions. Results generally showed that humidity had insignificant effect on the nanoscale friction at superhydrophobic DLC surface. The effect of the superhydrophobic double roughening DLC and friction were discussed with the following factors; the surface morphology affinity to needle-like shape, a reduction of the real area of contact, graphitization and easily occur to slip at small contact interface due to superhydrophobicity.
\end{abstract}

Key words: Superhydrophobic DLC, Nanoscale Friction, Humidity

\section{Introduction}

Diamond-like carbon (DLC) films are of considerable interest for a protective layer on the micro/nano-electromechanical systems (MEMS/NEMS). Generally DLC surface is automatically smooth and excellent mechanical properties of high elastic modulus and hardness with low friction coefficient. However, flat surface does not have good tribological properties, such as higher adhesion and friction force due to real area of contact increased with the humidity or various surface forces in nanoscale tribology. In addition, application of DLC film is limited due to its sensitivity to the environmental conditions, especially relatively humidity. The friction coefficient could continuously rise up to be high humid, which means that the formation of capillary bridges or a water meniscus between the AFM tip and the DLC surface. This meniscus layer, significant increase in the friction force as a driving force is requiring overcoming the meniscus induced adhesion force. And hence its surface needs to be fabricated chemically or topographically to enhance its tribological performance. Various modification methods have been proposed to explain the environmental dependence of the nanoscale frictional behavior of various films ${ }^{(1 \sim 3)}$.

The surface wettability has been also considered as one of the key parameters to affect

*Received 28 July, 2009 (№. 09-0381) [DOI: 10.1299/jamdsm.4.336]

Copyright $@ 2010$ by JSME 
the nanoscale frictional behavior of a humidity protecting thin film such as superhydrophobic DLC film. In superhydrophobic surface, it was reported that the water repellency and non-wetting could reduce friction and adhesion force at sliding interface. A surface is hydrophobic if the value of the wetting angle of water is over than $90^{\circ}$, the surface is superhydrophobic if the value of the wetting angle of water between $150^{\circ}$ to $170^{\circ}$. Especially, nature plant surface are combination of micro and nonometer scale of double roughness patterns, fractal roughness may lead to superhydrophobicity and easily gains wetting angle of $160^{\circ}$ with low hysteresis using paraffinic wax of $\mathrm{CH}_{2}$ groups ${ }^{(4)}$.

Recently B. Bhushan et al. ${ }^{(5 \sim 6)}$ have investigated the frictional behaviors of the lotus leaf with surface roughness and superhydrophobicity. They reported that the lotus and taro plant could be reduced the real area of contact between the surface, counterparts and environment, resulting in the minimum friction coefficient in ambient air.

In this research, we focused on the humidity independence of the nanoscale friction behavior of DLC film with double roughening structure. The double roughening structure was prepared by DLC film deposition on the Si (100) wafer with nanoscale Ni doted surface. Nanoscale friction behaviors were explored under humidity conditions and wetting angle measurements. Experimental details for fabrication of the double roughening DLC films and friction test under humidity environment were provided as blow.

\section{Experimental}

\subsection{Film preparation}

Surface modified of DLC include double roughening morphology on undulated DLC film ${ }^{(7)}$, fabricated by the process of $2.45 \mathrm{GHz}$ surface wave-excited plasma (SWP) CVD with the mixture of methane $\left(\mathrm{CH}_{4}\right)$ and tetramethylsilane (TMS: $\left.\mathrm{Si}\left(\mathrm{CH}_{3}\right)_{4}\right)$ gases. The films were deposited at a negative bias voltage of $0 \mathrm{~V}$, deposition pressure of $220 \mathrm{~Pa}$ and microwave power of $300 \mathrm{~W}$. Methane $\left(\mathrm{CH}_{4}\right)$ gas was used for a making undulated DLC film on nanoscale Ni doted surface.

Double roughening DLC film was coated on undulated DLC surface by a series of surface etching, coating process and surface etching. The etching process was performed for $1 \mathrm{~min}$ to modify the undulated surface. Ar plasma etching was induced the surface at $40 \mathrm{~Pa}$, $300 \mathrm{~W}$ for $1 \mathrm{~min}$, respectively. After etching process, the $\mathrm{CH}_{4}$ and TMS were used to deposition during $1 \mathrm{~min}$. The gas flow rates of $\mathrm{Ar}, \mathrm{CH}_{4}$ and TMS were followed conditions; 15:5:1 sccm. And then, Ar plasma etching was re-induced with a same condition.

\subsection{Film characterization}

Surface and cross-section morphologies of the films were observed field emission scanning electron microscopy (FE-SEM, Hitachi S-4300), the variation of roughness, width and height were analyzed by atomic force microscopy (AFM, Seiko SPA 400). Raman analysis was conducted for both flat DLC and the modified DLC films. The Raman spectrum was recorded in the range between 200 and $2000 \mathrm{~cm}^{-1}$. The Fourier transform infrared (FT-IR 6100, JASCO) measurements were carried out with a single beam spectrometer. The wetting angle measurements of each sample were performed using a contact anglemeter (Keyence, goniometer type) that analyzes the image of a sessile droplet on the surface. Water volume was $0.4 \mu \mathrm{l}$, each measured was repeated for 7 times and the average values were used.

\subsection{Measurement of nanoscale friction with various humidity conditions}

Nanoscale friction characteristic was evaluated with atomic/friction force microscopy (AFM/FFM, Seiko SPA 400) in different humidity conditions $\left(23^{\circ}\right.$ C, R.H. $\left.0 \sim 60 \%\right)$ over scan area of $3 \times 3 \mu^{2}$, scan rate of $0.05 \mathrm{~Hz}$ and normal force was $100 \mathrm{nN}$. The $0 \%$ of humidity was heated on plate at $80{ }^{\circ} \mathrm{C}$ for $15 \mathrm{~min}$. The $\mathrm{Si}_{3} \mathrm{~N}_{4}$ tip with $20 \mathrm{~nm}$ radius of 
curvature and normal spring constant $\left(\mathrm{K}_{\mathrm{z}}\right)$ was $0.75 \mathrm{~N} / \mathrm{m}$, and the results were compared to those of flat DLC, Si (100) wafer and an undulated DLC surfaces. The friction force, direction of lateral spring constant was calculated with Ref. 8 .

\section{Results and discussion}

\subsection{Topographical modification of superhydrophobic double roughening DLC}

Figure 1 shows the surface microstructures prepared in the present work. When using $\mathrm{CH}_{4}$ and Ar gases during DLC deposition at the pressure $220 \mathrm{~Pa}$, the doted surface is quite surface undulation as shown in Fig. 1 (a). Average heights, width of undulated DLC surface were $62 \mathrm{~nm}$ and $494 \mathrm{~nm}$. On the other hand, plasma etching with pure $\mathrm{Ar}$ gas, $\mathrm{CH}_{4}$ and TMS gases resulted in the deposited surface fabricated of double roughening structure such as needle-like sharp posts as shown in Fig. 1 (b) and (c). Height and width of the posts were measured to be $210 \mathrm{~nm}, 1080 \mathrm{~nm}$. Geometrical parameters of the nanoscale posts slightly increased as the deposition process. Furthermore, number density and asperity shape of top posts slightly changed due to high working pressure increased to $220 \mathrm{~Pa}$ and repeated etching process at $30 \mathrm{~Pa}$.

Figure 1 (d) shows the morphology of the needle-like sharp posts directly fabricated on bare $\mathrm{Si}(100)$ prepared by the same deposition process without undulated surface. For this approach, hydrophobic DLC coating on this Si (100) surface would modify the lotus and taro leaf that can be characterized by double roughening surface with additional layer of epicuticular wax coating on the surface. When high working pressure at $220 \mathrm{~Pa}$ and TMS gas mixture, it has a profound effect on nanoscale post generation because of large TMS particle is flow inner chamber. On the other hand, working pressure with $\mathrm{CH}_{4}$ and TMS gas reducing to $30 \mathrm{~Pa}$, surface was quite smoothed as shown in Fig. 2.

This result implied that there could be three important factors that affected the double roughening DLC surface. They are Ar plasma etching, high working pressure and TMS precursor gas. In addition, this approach will be increases in air pockets easily trap among the needle-like posts with liquid-air-solid contact such as Cassie and Baxter's model ${ }^{(9)}$ due to double roughening benefit from air pockets formation.
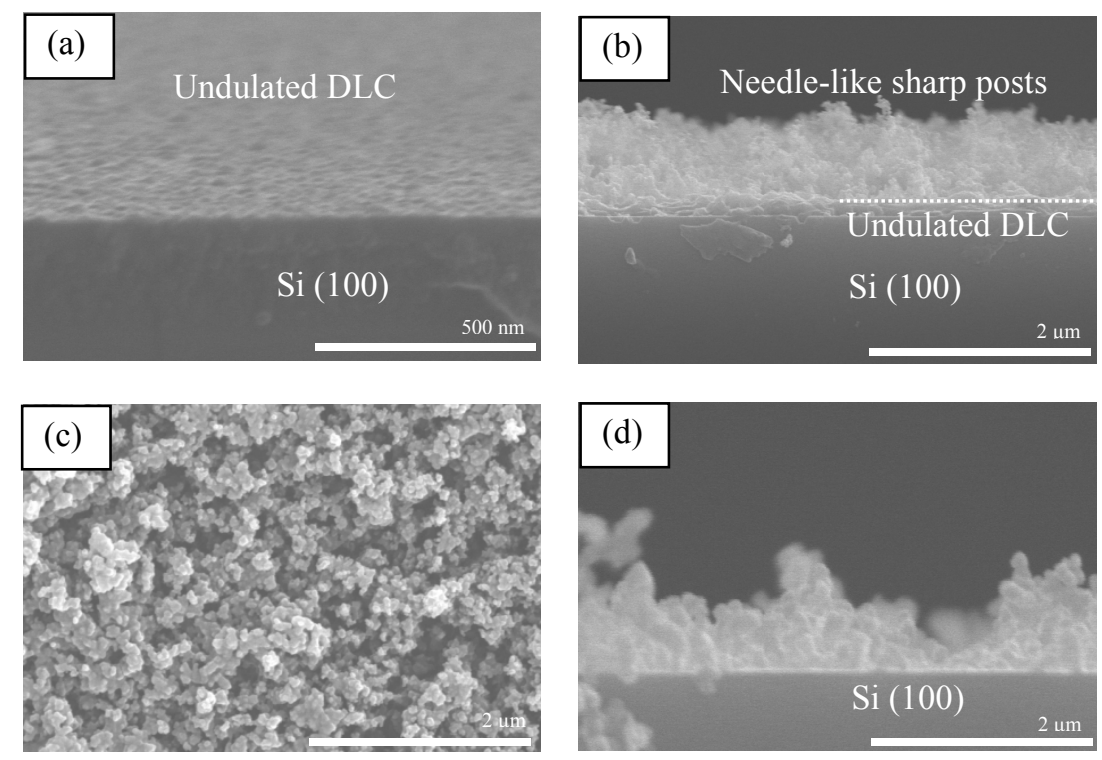

Fig. 1 FE-SEM images show the modify morphology of the DLC surfaces each step for the deposited at $220 \mathrm{~Pa}$, $300 \mathrm{~W}$ for 60s. (a) Undulated DLC of Ar, $\mathrm{CH}_{4}$ with Ni doted on the surface, (b), (c) Double roughening DLC of Ar, $\mathrm{CH}_{4}$ and TMS mixed, (d) Nanoscale posts generation of Ar plasma etching and deposited of TMS, Ar with Si (100) wafer on the surface. 

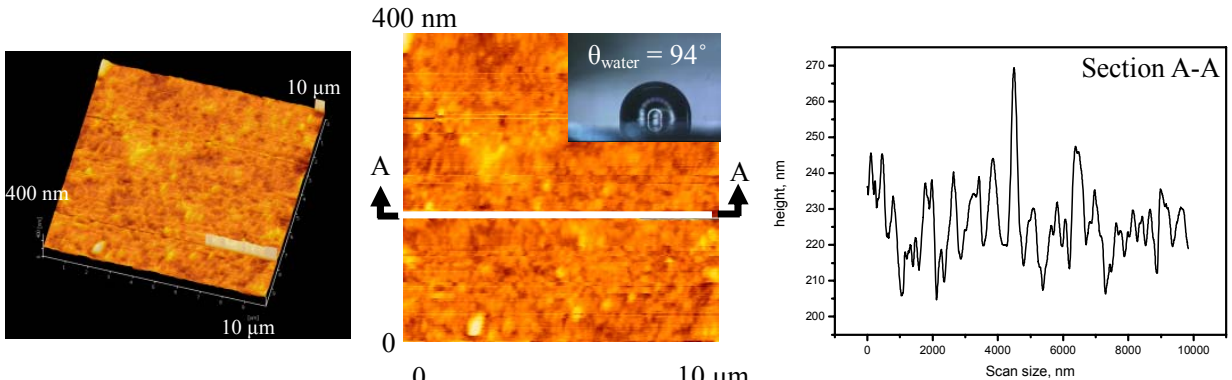

Fig. 2 Surface height map, 2D profile and corresponding wetting angle $\left(\theta_{\text {water }}=94^{\circ}\right)$ showing the flat DLC surface deposited for $\mathrm{Ar}, \mathrm{CH}_{4}$ and TMS precursor gases at $30 \mathrm{~Pa}$.

(a)

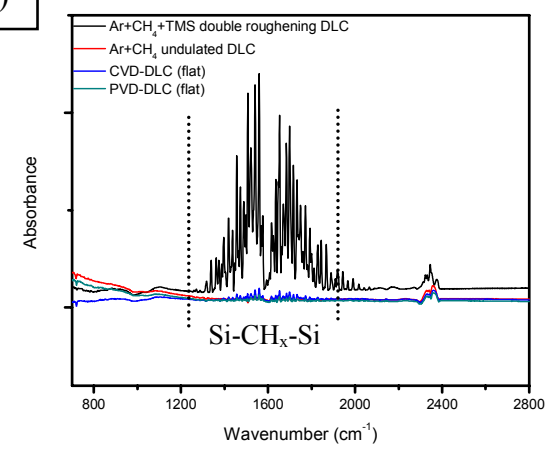

(b)

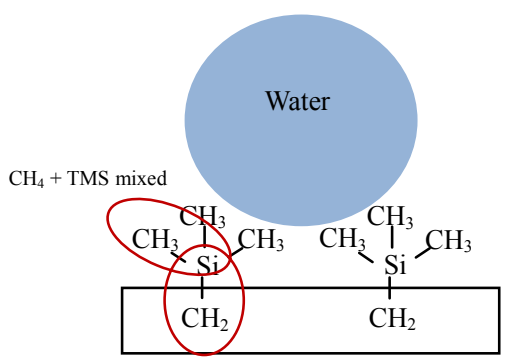

Fig. 3 (a) FTIR spectra for the superhydrophobic double roughening DLC with TMS and without contained DLC.

(b) A schematic view of the chemical model supported mixed $\mathrm{CH}_{4}$ and TMS precursor gases.

\subsection{Chemicals modification of superhydrophobic double roughening DLC}

The Fourier transform infrared (FTIR) analysis is widely used to characterize the bonding properties in DLC surface. Figure 3 shows the spectra for the superhydrophobic double roughening DLC with TMS and $\mathrm{CH}_{4}$ respectively, and is compare to that of flat DLC surfaces deposited by PVD, CVD and undulated DLC surface.

In case of superhydrophobic double roughening DLC film, it is appeared to the strong absorption bands at $1258-1890 \mathrm{~cm}^{-1}$ are assigned to the $\mathrm{Si}-\mathrm{CH}_{\mathrm{x}}-\mathrm{Si}$ stretching mode. In the present case, the relative intensity of the $\mathrm{Si}-\mathrm{CH}_{\mathrm{x}}-\mathrm{Si}$ increases with an increase in the TMS content, suggesting that the film with a higher hydrophobic $\mathrm{CH}_{3}$ content include more than others. It means that as the number of alkyl groups in the TMS precursor gas contained, therefore $\mathrm{Si}_{-} \mathrm{CH}_{\mathrm{x}}-\mathrm{Si}$ band indicating in a FTIR spectra. The results of TMS surface modification clearly shows that at least one functional groups covering the DLC surface. These facts are also seen to be reflected the static wetting angle in Fig. 5. The wetting angle of water increases with increase in the number of alkyl groups present in the TMS gas.

\subsection{Surface wettability and RMS roughness characteristics of DLC surface}

Figure 4 shows AFM images of the hydrophobic undulated DLC surface and the superhydrophobic double roughening DLC surface. A 3D topographical image clearly revealed the film's surface consisted of undulation and needle-like sharp posts. The size of each protrusion was approximately the same size as each segment observed in the $2 \mathrm{D}$ images. The RMS roughness of undulated DLC was $15.07 \mathrm{~nm}$. The static wetting angle of undulated DLC surface is $106^{\circ}$ as shown in Fig. 5 (a). In case of general flat DLC film, wetting angle was reported on $72 \sim 76^{\circ}$ the typical value of DLC ${ }^{(10)}$. It implies that the surface undulation acting between the water and the surface affected on the wettability. Figure 5 (b) is the water drop on the surface of double roughening DLC surface. Nanoscale 
of needle-like posts were increased the wetting angle to $161^{\circ}$. This film has the RMS roughness of $31.19 \mathrm{~nm}$ with $\mathrm{CH}_{3}$ of hydrophobic property. Figure 5 (c) shows the water drop on the surface of directly fabricated on the $\mathrm{Si}(100)$ wafer and the wetting angle was $132^{\circ}$. It can be generally also said that the nanoscale roughness on top of the posts was supports to the lotus effect. It is suggested that the undulated DLC film itself is hydrophobicity and the combination of $\mathrm{CH}_{3}$ property and the roughness of the double roughening is what create such as superhydrophobic surface.
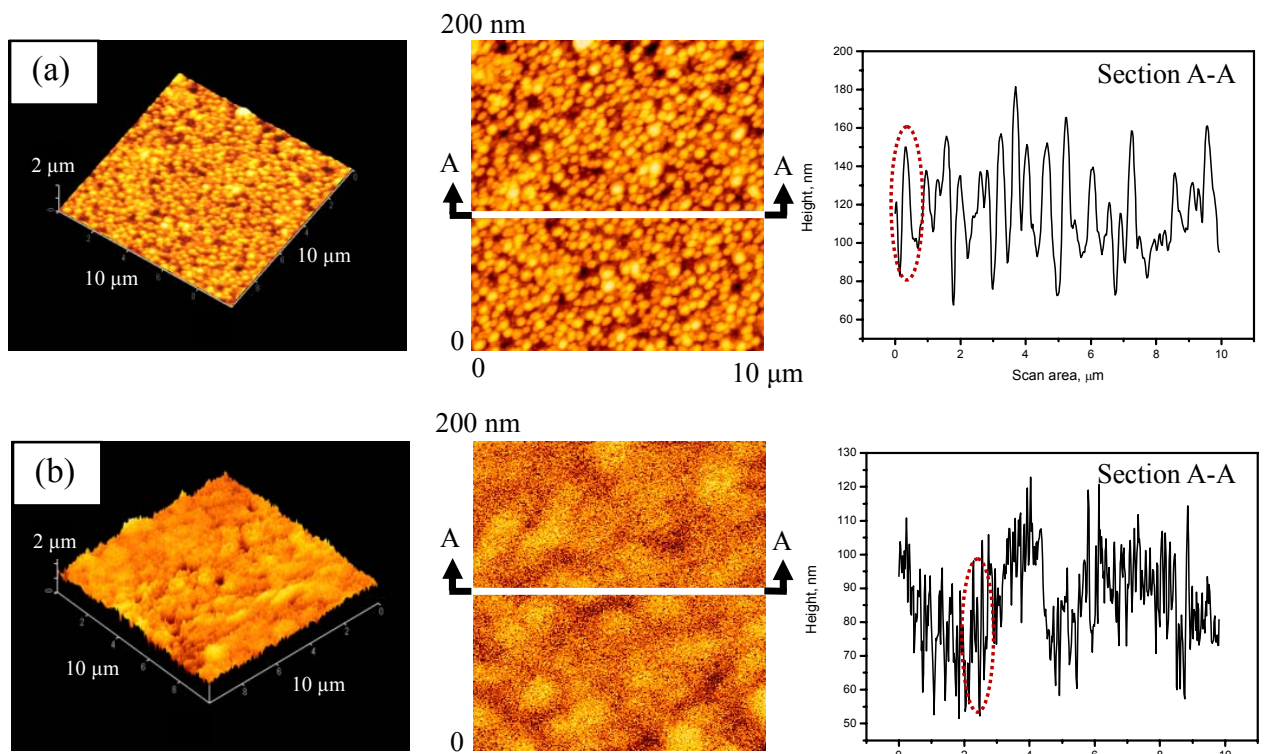

$200 \mathrm{~nm}$
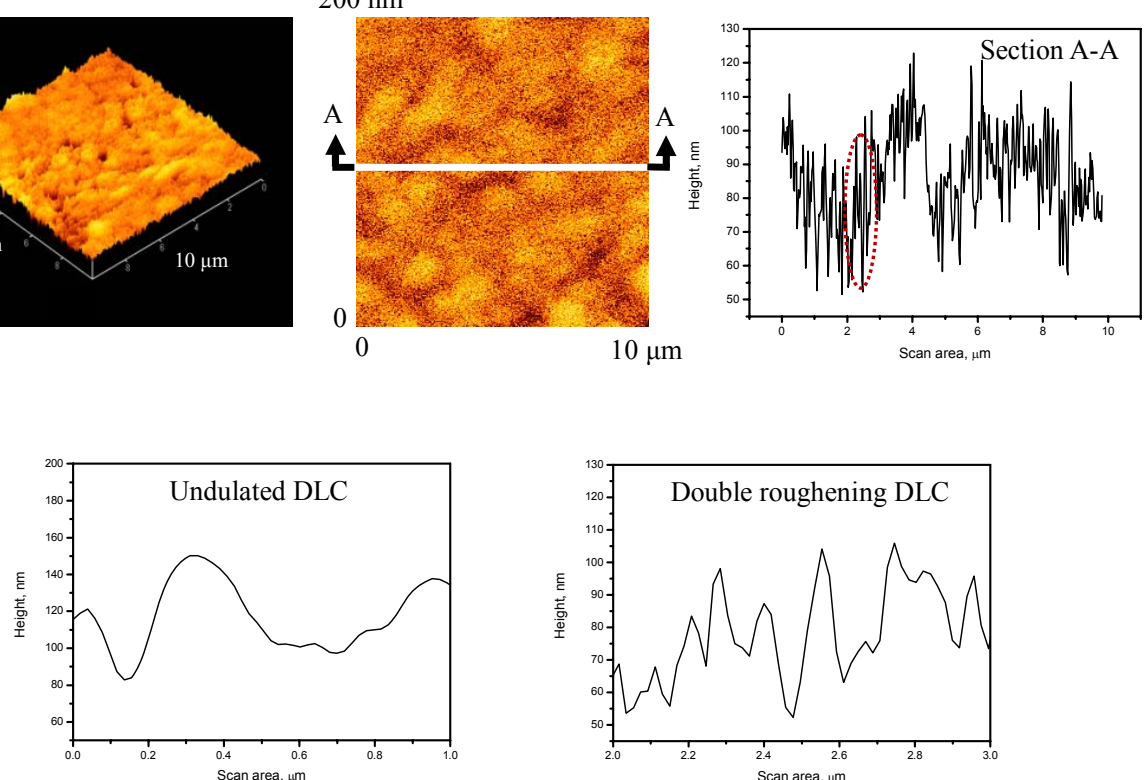

Fig. 4 Surface height maps and 2D profile showing the two kinds of DLC surfaces curve for two kinds of rough DLC surface using an AFM. The radius curvature is calculated from the parabolic curve fit.

(a) Hydrophobic undulated DLC surface, (b) Super-hydrophobic double roughening DLC surface.
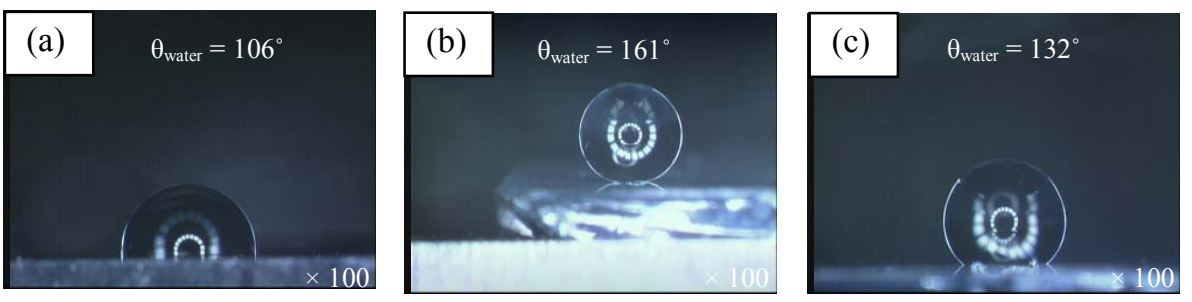

Fig. 5 Water droplet images on the modified surface with structure of (a) undulated DLC, (b) double roughening DLC and (c) needle-like posts directly fabricated on the Si (100).

\subsection{Raman analysis}

In Figure 6 (a), we show the Raman spectra of the above four samples. The Raman D and $\mathrm{G}$ peak, centered at approximately $1350 \mathrm{~cm}^{-1}$ and $1500 \mathrm{~cm}^{-1}$, respectively, composed the carbon peak ${ }^{(11)}$. Figure $6(b)$ shows the ratio of $\mathrm{I}_{\mathrm{D}} / \mathrm{I}_{\mathrm{G}}$ and variation of $\mathrm{G}$ peak position with 
different surface layer. The increase of the $\mathrm{D}$ peak content is an indication on the state of development of the $\mathrm{sp}^{2}$ phase and that the $\mathrm{sp}^{2}$ sites are beginning to organize into small graphitic clusters. Thus, increase of $\mathrm{I}_{\mathrm{D}} / \mathrm{I}_{\mathrm{G}}$ ratio indicates a decrease of the $\mathrm{sp}^{3}$ content. The integrated intensity ratio of $\mathrm{D}$ and $\mathrm{G}$ band was as high as 0.95 , which was comparable to that of ordered other sample, and this indicated high graphitization of double roughening structure of superhydrophobic DLC film.

As a series of surface etching, coating and surface etching process increased, which was comparable to that of carbon structure, and this indicated high graphitization of superhydrophobic coating. Raman analysis could confirm the graphitization of double roughening surface was also effected on the nanoscale friction behaviors.
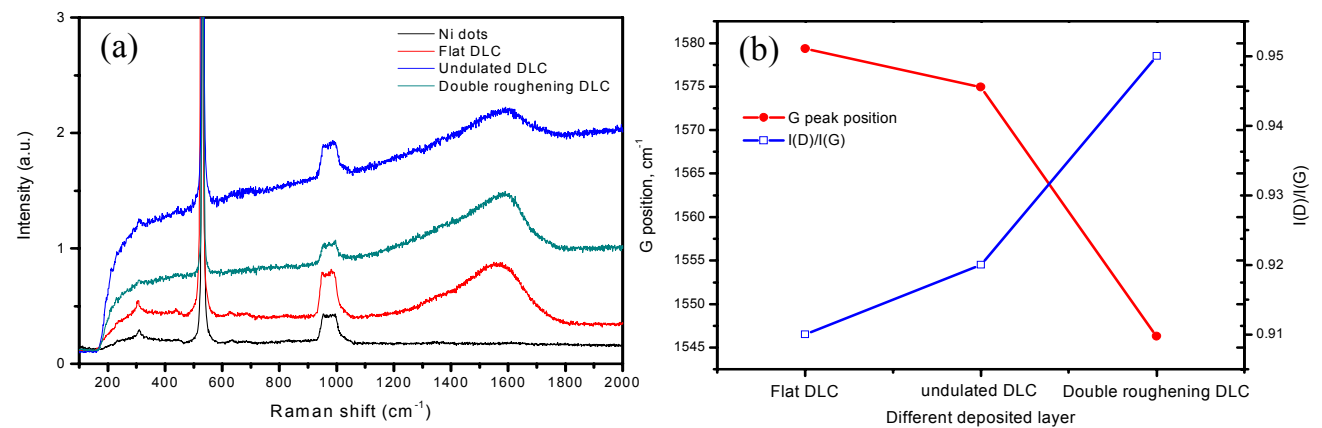

Fig. 6 (a) Raman spectra of DLC films as a function of deposition process, (b) $\mathrm{I}_{\mathrm{D}} / \mathrm{I}_{\mathrm{G}}$ ratio and G-peak positions extracted from Raman data.

\subsection{Nanoscale friction of double roughening DLC surface under different humidity conditions}

Generally, nanoscale friction behavior of a material could be affected by surface wettability which is necessary for superhydrophobiciy in most sliding contacts. Especially, adhesion and friction were increased with humidity due to meniscus at the contact interface. Figure 7 shows the characteristics of five kinds of surfaces with $\mathrm{Si}_{3} \mathrm{~N}_{4}$ tip radii of $20 \mathrm{~nm}$ under an applied normal load of $100 \mathrm{nN}$ and a relative humidity of 0 to $60 \%$. The nanoscale friction increased almost linearly up to $30 \sim 40 \%$ for all flat and undulated DLC surfaces and then it shows decreased with increasing humidity. The undulated surface has bigger friction than any other surface till the $50 \%$, and then decreased. It revealed that Si (100) wafer and flat DLCs were easily absorb water molecule in ambient air because of their hydrophilicity. However these hydrophilic characteristic was made of water lubricant soft tribo-film with increasing humidity, and then reduced friction. This result is consistent with Bhushan's result ${ }^{(12)}$.

Double roughening DLC film showed constantly lowest friction than among the flat films. It was thought that the self-lubrication caused by a superhydrophobic property was mainly responsible for this friction. Therefore, friction decreased as the wetting angle of water increased, i.e. as the surface wettability changed in nanoscale friction. If the existence of a water molecule, such as a lubricant or absorbed water at the interface between two hydrophilic parts during sliding contact due to high surface energy, which increases friction. However, for the friction values there is a constantly and lowest when the double roughening DLC film because the tip sliding between the needle-like asperities results in easily occur to slip at small contact interface. It was found that humidity had insignificant effect on the nanoscale friction, and superhydrophobicity a major component of the friction force experienced by the tip.

Furthermore, a characteristic of double roughening structure ensures that hydrophobicity with sharp posts and real area of contact at small interface between tip and surface induced 
may also lead to a very low friction with a liquid slip. According to Hertzian and JKR model ${ }^{(13)}$, the difference in real area of contact among the DLC samples are compared at table 1. According to the JKR theory, the real area of contact of the double roughening DLC film was lower than other DLCs, which is due to their lower contact areas when compared to samples. The modified surfaces have a higher wetting angle of water values when compare to PVD, CVD DLC and Si (100) wafer and thus would have a lower surface energy. Lower surface energy would therefore result in lower values of real area of contact, which relatively corresponding results in lower values of friction. From Fig. 4, it can be observed that the contact area decreased with the changed morphology. This explains well the increased in friction force with the humidity for both Si wafer, PVD and CVD flat DLC surface, which is due to the increase in the real area of contact.

In addition, the graphitization of double roughening DLC was also reduced parameter of friction, which was comparable to that of Raman spectrum as shown in Fig. 6.

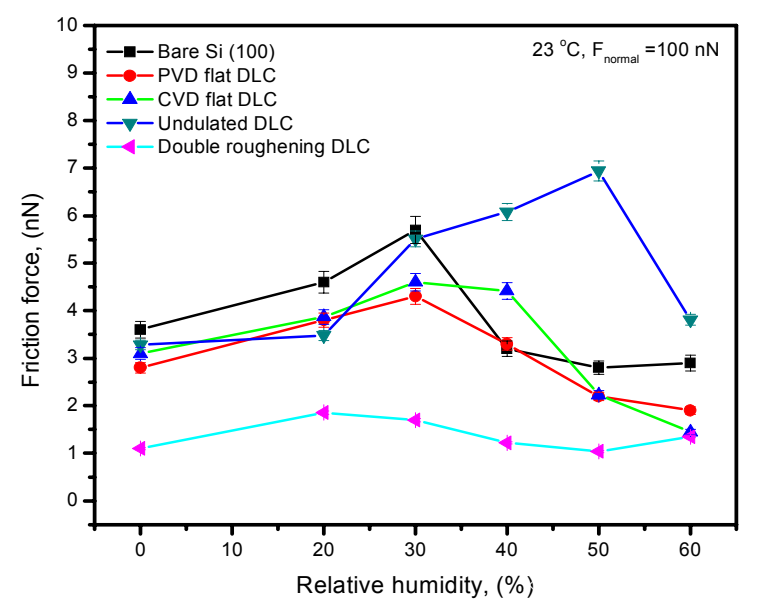

Fig. 7 Influence of relative humidity (R.H.) on the friction force of Si (100) wafer, CVD DLC, PVD DLC, undulated DLC and double roughening DLC at $100 \mathrm{nN}$ in air conditions.

Table 1 Real area of contact calculated Hertz and JKR model, $\mathrm{A}_{\mathrm{r}}=\mathrm{nm}$

\begin{tabular}{lccccc}
\hline \multicolumn{1}{c}{ Material } & Hertz model, $\mathrm{A}_{\mathrm{r}}$ & JKR model, $\mathrm{A}_{\mathrm{r}}$ & $\begin{array}{c}\text { Young's } \\
\text { modulus }(\mathrm{GPa})\end{array}$ & $\begin{array}{c}\text { Poisson's } \\
\text { ratio }\end{array}$ & $\begin{array}{c}\text { wetting } \\
\text { angle }\left({ }^{\circ}\right)\end{array}$ \\
PVD DLC & 2.16 & 17.23 & 195 & 0.18 & 72 \\
CVD DLC & 2.19 & 18.48 & 193 & 0.18 & 67 \\
Undulated DLC & 1.16 & 2.11 & 184 & 0.18 & 96 \\
Double roughening DLC & 0.96 & 1.95 & 104 & 0.18 & 161 \\
\hline
\end{tabular}

\section{Conclusions}

In this research, we have characterized the humidity independence of the nanoscale friction behavior of DLC film with double roughening structure. Superhydrophobic double roughening DLC film prepared by the $2.45 \mathrm{GHz}$ surface-wave excited plasma CVD method made it possible to investigate the nanoscale frictional behaviors of the DLC film with various surface morphologies such as flat DLC, undulated DLC and double roughening DLC against $\mathrm{Si}_{3} \mathrm{~N}_{4}$ tip under the differently humidity. The following are the conclusions that could be drawn from the present work on the tribological behavior of superhydrophobic double roughening DLC.

(1) A superhydrophobic and self-lubricating DLC surface $\left(\theta\right.$ was $\left.161^{\circ}\right)$ was successfully fabricated on the undulated DLC film by geometrical and chemical 
modification. It can be seen that the combination of double roughening morphology and the number of alkyl groups in the TMS precursor gas, therefore static wetting angle of water increases with alkyl groups spread surface as $\mathrm{Si}_{-} \mathrm{CH}_{3}$ property.

(2) Double roughening DLC film showed constantly lowest friction than among the flat films. It was thought that the self-lubrication caused by a superhydrophobic property was mainly responsible for this friction. It was found that humidity had insignificant effect on the nanoscale friction.

(3) As a series of surface etching, coating and surface etching process increased, the surface was changed with graphite-like structures. This result shows that the graphitization of double roughening surface was also effected on the nanoscale friction behavior.

\section{References}

(1) L. Wang, S. Guo, S. Dong, Electrochemistry communication Vol. 10, (2008), pp. 655-658.

(2) E.S. Yoon, S.H Yang, H.G. Han, H. K, Wear, Vol. 254, (2003), pp. 974-980.

(3) Y.C. Jung, B. Bhushan, Scripa materialla Vol. 57 (2007), pp.1057-1060.

(4) Ma, M., Hill, R., Current Opinion in Colloid and Interface science Vol.11, (2006) pp. 193-202.

(5) Z. Burton, B. Bhushan, Ultramicroscopy Vol.106, (2006), pp. 709-719.

(6) M. Nosonovsky, B. Bhushan, Material Science and Engineering R 58, (2007) pp. 162-193.

(7) Y.J. Jang, N. Umehara, Tribology Online Vol.3, (2008), pp. 294-297.

(8) G.Meyer, N.M. Amer, Apply Physics Letter Vol. 57, (1990), pp. 2089-2091.

(9) E. Bormashenko, Y. Bormashenko, T. Stein, G. Whyman, E. Bormashenko, Journal of Colloid and Interface Science Vol.311, (2007), pp. 212-216.

(10) Y.J. Jang, H. Kousaka, N. Umehara, Proceeding of the International Tribology Conference, Nagoya, (2008), pp. 497-498.

(11) M. Yoshikawa, Materials Science Forum 52-53 (1980), pp. 365-386.

(12) H. Liu, B. Bhushan, Ultramicroscopy, Vol.97, (2003), pp. 321-340.

(13) K.L. Jonson, K. Kendall, Proceeding of the Royal Society of London, Series A, 324, (1971), pp. 301-313. 